\title{
Why Acute Inflammatory Response Can Happen After Adenoviral Vector Vaccines; Is the Interleukin 6 the Secret and How Circumvent that, Is by Adding Polyethylene Glycol (PEG)?
}

\section{Amr Ahmed ${ }^{1 *}$, Hassan A Shora ${ }^{2}$, Ahmed Mohamed $^{3}$ and Simona Ibrahim $^{4}$}

${ }^{1}$ Department of Public Health, Tuberculosis Program, First Health Cluster, Ministry

of Health, Riyadh, Saudi Arabia

${ }^{2}$ Department of Molecular Biology/Biochemistry, Port-Said University, Egypt

${ }^{3}$ Faculty of Dentistry, the British University in Egypt, Cairo, Egypt

${ }^{4}$ Department of Pharmaceutical Chemistry, Faculty of Pharmacy, the British

University in Egypt, Cairo, Egypt

*Corresponding Author: Amr Ahmed, Department of Public Health, Tuberculosis

Program, First Health Cluster, Ministry of Health, Riyadh, Saudi Arabia.
Received: April 15, 2021

Published: June 19, 2021

(C) All rights are reserved by Amr Ahmed., et al.

\section{Abstract}

After one year of pandemic SARS COV2, which started in December 2019, it is still considered a health disaster in all nations. Despite vaccination efforts, the number of infected people increased, also the number of SARS COV2 variants increased. Certain vaccinations are approved under Emergency Use Authorization, including those dependent on mRNA technology, like those developed by Pfizer-BioNTech and Moderna (FDA Emergency Use Authorization EUA), as well as those dependent on Adenovirus vectors, like those developed by AstraZeneca (covishield, AZD1222 vaccine. (ChAdOx1) (WHO permission) and Johnson and Johnson, (FDA suspended 13/4/2021 use FDA and CDC suggested the stop use of COVID-19 (Janssen \& Janssen) vaccination when reviewing details of six U.S. case-rare and serious blood clot cases identified in vaccine-receiving individuals). The optimal vaccine will have a low sero prevalence of neutralizing antibodies against the viral vector and a low incidence of adverse events. The protection of non-human adenovirus vectors used in humans is a primary concern. Global efforts against covid-19 continue, and vaccine production has intensified. Adenoviral vector vaccines induce an acute immunogenic response that varies according to the immune status of the individuals injected. The excipient used in the vaccine injection can either increase or decrease the severity of this acute immune response. For example, polyethylene glycol can decrease IL-6 while polysorbate 80 or EDTA can increase severity. In comparison to Americans or Asians, Africans have neutralizing antibodies against the chimpanzee adenoviral vector, which can result in a less acute immunogenic response (less adverse effects). Thus, it is necessary to administer ChAdox1 nCov2 to African populations in order to avoid the vaccine's acute immunogenic impact in neutralizing antibodies existence (vaccine less effective). The question after discussing the acute immune response from adenovirus vectors (IL-6 is has a role) and Is adding polyethylene glycol to adenoviral vector vaccine lessen the vaccine adverse effects.

Keywords: Covid-19 Vaccine; Adenoviral Vector Vaccines; Thrombocytopenia; EDTA; mRNA Vaccine; Inflammatory Response; IL-6; Polyethylene Glycol (PEG); Polysorbate 80; African People; Zinc 
Why Acute Inflammatory Response Can Happen After Adenoviral Vector Vaccines; Is the Interleukin 6 the Secret and How Circumvent that, Is by Adding Polyethylene Glycol (PEG)?

\section{Introduction}

After one year of pandemic SARS COV2, which started in December 2019, it is still considered a health disaster in all nations. Despite vaccination efforts, the number of infected people increased, also the number of SARS COV2 variants increased. Certain vaccinations are approved under Emergency Use Authorization, including those dependent on mRNA technology, like those developed by Pfizer-BioNTech and Moderna (FDA Emergency Use Authorization EUA), as well as those dependent on Adenovirus vectors, like those developed by AstraZeneca (covishield, AZD1222 vaccine. (ChAdOx1) (WHO permission) and Johnson and Johnson, (FDA suspended 13/4/2021 use FDA and CDC suggested the stop use of COVID-19 (Janssen \& Janssen) vaccination when reviewing details of six U.S. case-rare and serious blood clot cases identified in vaccinereceiving individuals). The optimal vaccine will have a low sero prevalence of neutralizing antibodies against the viral vector and a low incidence of adverse events. The protection of non-human adenovirus vectors used in humans is a primary concern.

In spite of the fact that majority of non-human adenovirus vectors are replication-deficient in human cells and remove of important genes causes viral replication blunting through viral gene expression, examination and the persistence of viral genome in a diversity of human cells and in a non-human primate design will be needful to determine the protection of these vectors [1].

Because of their greater transgenic efficacy as compared to other forms of transgenic, viral vectors appear to be among the most advantageous transgenic carriers for gene therapy. At the moment, AdV vectors are used in $23.5 \%$ of human research because of its capacity to activate a large quiescent cell and divide them effectively. They seldom integrate into the host genome, which significantly reduces the insertional mutagenesis risk [2].

About $80 \%$ of older individuals were subjected to such HAdV serotypes normally [3]. An extreme immune response to AdV vectors may have devastating consequences in humans, as demonstrated via a serious reason of systemic inflammatory response condition primarily due to the AdV transgenic vector delivered systemically [4]. To decrease these immune responses, it has been proposed to eliminate the epitopes and liver tropism involved in viral protein recognition through antibody neutralization. By exploiting the AdV capsid protein isoforms diversity and their ligand-receptor inter- actions, vectors that depend on low seroprevalent AdVs could be used to target a range of cellular forms in addition to overriding preexisting immunity. In all human species, non-human AdV has a naturally low seroprevalence since their creation in the 1990s [5].

Leading non-human adenovirus candidates to involve vectors originated from simian adenoviruses (SAds), most notably chimp adenovirus (termed ChAds or AdCs). We recently identified the production of ChAdOx1, a chimeric vaccine vector, from a simian adenovirus (ChAd) serotype Y25. This was achieved through red recombination engineering, in which the native genes $\mathrm{E} 4$ or $\mathrm{f} 4$, or f6 and or f6/7 were replaced with HAdV-C5 (human adenovirus). The previously described vector demonstrated a raise in hexon protein synthesis from HEK293 cells in comparison to the ChAd parent viruses and ChAdOx2. Additionally, the deleted vaccine vector E1/E3-, that is derived from ChAd68 and contains an adjusted E4 area, increased virus yields in HEK293 cells. SAd viral vaccines are viruses with E1-E3 deletions. Due to the requirement that the vaccine vector is non-replicating and the fact that adenovirus genes have limited immunomodulatory activity, The E1 region, which encodes viral transactivator proteins required for virus development, and the E3 region, which encodes immunomodulatory proteins, are absent from sad vectors. Antibodies targeted against the vector greatly decrease the fundamental immune response to the transgene material that can compromise the vaccine vector's efficacy. These findings demonstrate the critical importance of considering the population's immune status to be vaccinated while developing a vaccine vector [6].

Specific SAdV-based vectors as vaccines can be ineffective in specific cases due to the detection of neutralizing antibodies to several chimp adenovirus serotypes in individuals from Brazil, China, and Sub-Saharan Africa. Individual sera from Sub-Saharan Africa, Thailand, and United States were evaluated for antibodies neutralizing three chimp adenoviruses and chimp sera. People from SubSaharan Africa had a higher prevalence of antibodies than individuals from Thailand or the United States. This result indicates that chimp adenoviruses are transmitted cross-species [7].

ChAdOx1 is directly associated with man adenoviruses of subgroup B2 and utilizes CD46 as the host cell receptor. CD46 is a supplement regulatory protein work on all human cells with the exception of erythrocytes [8]. 
Why Acute Inflammatory Response Can Happen After Adenoviral Vector Vaccines; Is the Interleukin 6 the Secret and How Circumvent that, Is by Adding Polyethylene Glycol (PEG)?

As a result, ChAd1 exhibits a wide inefficiency tropism, enabling it to infect cells and infection mechanisms that do not access other serotypes of Ad. Adenovirus vectors with low replication capacity are being utilized in early-stage human studies to treat a variety of disorders, like cancer, hepatitis C, HIV, malaria, and influenza [9].

The cell immunogenicity of the discarded ChAdY25 recombinant E1 E3 was linked to other E-derived chimpanzee adenovirus vectors, like ChAd 63, the first simian adenovirus vector to enter human studies. In the Gambian and British samples analyzed, the seroprevalence of vector neutralizing antibodies against ChAd Y25 was similar to or less than that subsequently believed for other chimp adenoviruses. As a result, we suggest that ChAdY25 may also be an effective vaccine in clinical studies [10].

Unlike the commonly used human adenovirus 5 vector, the ChAd0x1 vector is not impaired by major preexisting anti-vector immunity, restricting vaccine effectiveness in humans [11].

The AstraZeneca COVID-19 Vaccine is a replication-deficient adenovirus vaccine vectored in chimps (ChAdOx1-Chimpanzee Adenovirus Oxford1) [12].

Adenoviruses are extremely efficient vectors for triggering and enhancing the immune system to recombinant antigens encoded within them. ChAdOx1 Np+M1 is a novel recombinant simian adenovirus that has been used in the first dose-escalation test in humans by a $3+3$ study design. ChAdOx 1 is an effective vaccine vector which may be utilized to connect vaccine antigens in situations requiring robust cell immune responses for protection [13].

The ChAdOx1 vector virus is generated from the chimp's adenovirus Y25 and lacks the E1 and E3 genes. The seroprevalence of significant clinical neutralizing antibodies against Y25 in humans was calculated through unspecified adult serum specimens and was presented to be nil in young people in the United Kingdom ( $\mathrm{n}=$ 100) but 9 percent in adults in Gambian (n-57) [14].

Adenoviral vectors have several limitations due to their non-integrative nature, the fact also that genomes are produced from episomal DNA and are missing during cell division; furthermore, they could be cleaved even in non - dividing cells. As a consequence, adenoviral vectors are unfit for long-term expression in populations of cells that divide quickly. Additionally, the vectors induce acute inflammation, causing the release of pro-inflammatory cytokines like interleukins like tumor necrosis factor-alpha (TNF- $\alpha$ ), IL-8 and IL-6. Even so, individuals have died as a result of an acute reaction to adenoviral vectors that were injected. Further to decrease the immunogenicity of adenovirus vectors, we formed a helper-dependant vector system that includes only the therapeutic gene sequence of one virus (helper) and of the other virus (target). Inverted terminal repeats (ITRs) and the wrapping virus signal [15].

Polysorbate 80 and Polysorbate 20 are the most frequently occurring surfactants in biology. Surfactants are often utilized in the forming methods as raw resources, like filtration, lyophilization, transportation, storage and purification. Additionally, they are considered as a complete product to preserve proteins, avoid accumulation, and facilitate protein folding. Polysorbates, despite their ability to stabilize API, are vulnerable to oxidative degradation, indicating the presence of reactive oxygen species and residual peroxides. These byproducts were found to be more prevalent in a polysorbate 80-containing interleukin-2 formulation [16]. Consequently, polysorbates serve as photo stimulants, causing photooxidation. Multiple studies have shown that the surfactant's structure and composition have a significant impact on the photostability of antibodies [17].

In vitro, PEGylated HAdV vectors usage is due to reduced IL-6 production and lowered IL-6 and IL-12 production in vivo. Additionally, coated HAdV vectors because of declined serum IL-6 levels because of reduced spleen AdV intake).

In vivo, the use of PEGylated AdV vectors because of a decline in hepatic tropism and a decrease in spleen and liver toxicity. Additionally, proof suggests that AdV polymer coating approaches have the potential to overcome preexisting immunity to all viral capsid proteins). However, the recorded immunogenicity of PEG can limit the efficacy of PEGlyated AdVs [18].

Once adenoviral vectors are systemically introduced, they instantly stimulate innate immune responses, as demonstrated by the forming of coagulopathy, pro-inflammatory chemokines, thrombocytopenia, liver failure and cytokines [19]. The innate immune system is activated (TLRs) by the interaction of Ad vectors with intermediaries of complementary receptors, blood clotting factors, and toll-like receptors (TLRs). The Ad capsid interacts with 
Why Acute Inflammatory Response Can Happen After Adenoviral Vector Vaccines; Is the Interleukin 6 the Secret and How Circumvent that, Is by Adding Polyethylene Glycol (PEG)?

compounds of the typical, alternative, and classical complement systems in the inclusion or exclusion of preexisting antibodies, due to complement activation. Individuals who are Ad seropositive and reach a great dosage of Ad vectors are more likely to experience extreme complement activation, that can cause life-threatening systemic responses. The Ad vector's interaction with the blood clotting factors VIII(FVIII), protein c, FIX, and FX, and facilitates hepatocyte and Kupffer cell transduction. These pro-inflammatory chemokines and cytokines include interferon $\gamma$ and $\lambda$, tumor necrosis factor (TNF)- $\alpha$, interleukin-12 (IL-12), interleukin-8 (IL-8), and interleukin-6 (IL-6) [20].

Inoculation of a HAd vector resulted in thrombocytopenia due to platelet depletion in rhesus monkeys [21].

TNF- $\alpha$ and IL- 6 are essential mediators of the innate immune response that occurs in the presence of an Ad vector. We detected a significant reduction in pro-inflammatory cytokines, a decrease in cellular infiltrates in the liver, and a prolongation of transgene expression when TNF- $\alpha$ was inhibited via the Ad-encoded soluble TNF- $\alpha$ receptor [22].

PEG, which is FDA-approved, is often used to covalently couple therapeutic proteins. More than 18,000 PEG particles can be covalently linked to a single Ad particle, altering the main capsid proteins, pentons, fibers, and hexons, that are aims of anti-HAd5 neutralizing antibodies (Nabs). Pegylation of Ad vectors is an important method of overcoming high Nabs titers without sacrificing biological activity. This strategy results in decreased rates of Adspecific adaptive immune responses and prolongs transgene expression length, PEG is a synthetic linear polymer with a molecular weight of 200 - 40,000D. It is an extremely desirable compound for altering a variety of peptides or proteins due to its immunogenicity, low toxicity, and hydrophilicity. This has been shown to be true for adenovirus PEGylation as well. The most effective solvent has been determined to be PEG 20kDa. When compared to native Ad5, the rate of PEGylation was inversely related to immune activation and IL-6 expression [23].

Ad vectors activate innate immune responses immediately upon systemic administration, as evidenced by the development of proinflammatory cytokines and chemokines, liver damage, thrombocytopenia, and coagulopathy [24].
Individuals who are seropositive for Ad and receive a great dose of Ad vectors are at a greater issue of severe complement stimulation, resulting in life-threatening systemic responses [25].

Prior to PLGA encapsulation, PEGylation of Ad increased its stability and transduction performance while decreasing cytokine synthesis in vitro [26].

In the nonparenchymal liver cells and spleen, PEGylation decreased vector uptake. Additionally, PEGylation prevented the production of thrombocytopenia [27].

It'd be interesting to learn as much about the effects of alginate encapsulation on the innate immune response to Ad vectors. Alginate encapsulation can impact Ad tropism by interacting with blood factor-adrenergic receptor associations. The Ad particles are released slowly from the alginate microsphere, which minimizes liver toxicity [28].

Antibodies neutralizing Ad (AdC) of chimp source are not fund in the Asian and American individuals and are found in only trace amounts in the African population [29].

Adenoviral vectors lacking early transcript regions were found to be less responsive to IFN and inflammatory responses. Elimination of the E1 region resulted in a greater reduction in IFN resistance than deletion of the E4 region. Additionally, vectors deficient in E3 genes were more susceptible to eliciting an inflammatory response. It is worth noting that E2 transcripts haven't demonstrated to inhibit the innate immune response. Other than that, E2 gene transcripts stimulate the immune system [30].

\section{Discussion}

Global efforts against covid-19 continue, and vaccine production has intensified. To date, four vaccines have been designated in case of emergency, two of them were accepted by the FDA (PfizerBioNTech and Moderna), and one has been suspended by the FDA (Janssen and Janssen vaccine). PFIZER/BioNTech developed the first mRNA vaccine for human use, two adenovirus vector vaccines, one from the simian origin (AstraZeneca vaccine (AZD1222) (covishield)(ChAdOX1) and one from the human origin (Janssen and Janssen). 
Why Acute Inflammatory Response Can Happen After Adenoviral Vector Vaccines; Is the Interleukin 6 the Secret and How Circumvent that, Is by Adding Polyethylene Glycol (PEG)?

FDA regulations state darbepoetin alfa (polysorbate 80) has been shown in clinical trials to raise the harm of adverse effects, like death in some cases, stroke, heart failure, blood clots, and heart attack. Additionally, it has been demonstrated that it decreases overall survival and/or raise the likelihood of tumor growth or relapse in some kinds of patients with cancer) (access data. FDA).

Innate host immune reactions to adenovirus vectors and preexisting immunity to adenoviruses may cause toxicity and restrict gene transfer performance and time. While the effect of these host reactions can be reduced by concurrent immunosuppressive treatment, amendments to adenovirus vectors have been utilized to avoid host reactions and improve transgenic delivery performance. For instance, by modifying the adenovirus capsid covalently with synthetic polymers like polyethylene glycol, immunogenic epitopes could be protected, and immune system identification avoided [31].

The vectors enhance biphasic production of pro-inflammatory proteins such as TNF $\alpha$, IL-1 $\beta$, IL-12, IL-6, and IFN $\gamma$ and a variety of chemokines. The spleen produces IL-6, while Kupffer cells and dendritic cells produce the majority of the other proteins [32].

When replication-defective Adenovirus vectors are delivered systemically, they trigger more serious effects, like hepatic lesions suggested through increased transaminase levels, as well as thrombocytopenia and neutropenia [33]. The subsequent cytokine storm and robust innate immune response resulted in numerous organ failures and ultimately resulted in the patient's death. It is unknown why one patient's innate immune response was comparatively greater than that of the others. A quick memory response to the virus and genetic predisposition are two potential explanations.

EDTA is a highly effective zinc-chelating factor that is widely utilized in tests of protein interaction and molecular biology. $\mathrm{Zn}^{2+}$ is much more tightly bound to EDTA than other divalent metals like $\mathrm{Mg}^{2+}(\mathrm{kd}$, approximately $109 \mathrm{M})$ and $\mathrm{Ca}^{2+}(\mathrm{kd}$, approximately $1011 \mathrm{M}$ ). As a result, even in the existence of high levels of these other divalent cations, EDTA has the ability to drain a solution of free $\mathrm{Zn}^{2+}$ selectively [34].

Because of its versatility in counting and sizing blood cells, EDTA, also identified as a calcium chelator, is regarded as an effective and safe anticoagulant for a complete blood count. Platelet clumping, on the other hand, occurs infrequently. Individuals who have been diagnosed with cancer, chronic liver disease, infection, pregnancy, autoimmune disorders, or cardiovascular disease are at a raise risk of growing EDTA-dependent PTCP. Additionally, it has been detected in disease-free patients. In the presence of EDTA, platelets clump due to an autoantibody directed against the glycoprotein IIb/IIIa found on the platelet cell membrane [35].

While plasma zinc concentrations range between 10 and 20 $\mathrm{mM}$, zinc-binding to plasma proteins reduces free zinc to 0.5 - 1 mM.5 Zinc Platelet cytosol and alpha granules contain up to 60 times the amount of zinc found in plasma. 3.5 zinc as platelets are activated, zinc is emitted from them, raising the level of free zinc in the plasma and the microenvironment of a growing thrombus. Chelation of platelet zinc prevents phosphorylation of tyrosine kinase and platelet activation, implying that intracellular zinc contributes to platelet activity. Zinc stimulation led to increased platelet protein tyrosine phosphorylation, granule release, and secondary activation of IIb3, whereas zinc chelation inactivated phosphorylation and accumulation in response to numerous agonists.

This connection between accumulation and zinc-induced phosphorylation, as well as the impact of intracellular chelating agents, BAPTA-AM and TPEN is proportionate with zinc expending an intracellular impact, either directly or indirectly via stimulation of intracellular signaling mechanisms. Additionally, the complete inhibition of zinc-induced accumulation in the presence of PKC demonstrates that zinc-induced aggregation is dependent on intracellular biological processes. All these findings provide compelling evidence that zinc participates in platelet activation via an active, dynamic transmembrane signaling pathway [36].

There are novel findings demonstrate that IL-6 can prime platelets, predisposing them to activate the collagen receptor GPVI, resulting in platelet adhesion and thrombotic responses via modulating the expression of P-selectin (required for platelet activation and heterotypic and homotypic platelet interactions) and IIb3 receptor (associated in platelet aggregation and plug stabilization). These effects encourage platelets to adhere to and stabilize the platelet plug, predisposing platelets to aggregate in arterioles. During platelet activation, GPVI pools are redistributed (i.e., ultrastructural changes occur that result in an increase in GPVI on the activated platelet surface and a decrease in interior expression). 
Why Acute Inflammatory Response Can Happen After Adenoviral Vector Vaccines; Is the Interleukin 6 the Secret and How Circumvent that, Is by Adding Polyethylene Glycol (PEG)?

One might speculate that a critical implication of this molecular interaction is that it facilitates crosstalk between collagen-induced and inflammatory cytokine-induced GPVI pools [37].

Platelet factor 4 (CXCL4) is a chemokine that is emitted throughout platelet activation and in huge quantities in the vicinity of increasing blood clots. It helps to ensure the creation of layers that seal the clot, accelerates anticoagulant protein $\mathrm{C}$ activation, increases fibrin fiber polymerization, and significantly changes the fibrin networks morphology by docking PF4 to D-dimers in a tentative manner. Every PF4 can bind four D-dimers and every D-dimer can bind two PF4 in this hole b model, allowing for the construction of complex molecular edifices. Following PF4's long edge, D-dimers form an elongated helix that completes each seventh PF [38].

Pretreatment with EDTA eliminates the paracoagulant properties of zinc precipitated PF4 [39].

IL-6 also leads to hemostasis by platelet production, as Burstein elegantly summarized (1997). In the absence of other growth factors, IL-6 was shown to induce megakaryocyte maturation. Additionally, it can induce megakaryocyte proliferation. Additionally, IL-6 impairs platelet function and increases platelet activation caused by thrombin. Thus, by activating both primary and secondary hemostasis pathways. IL-6 tends to effect on production of unstable fibrin clots [40].

EDTA-induced thrombocytopenia (EDTA-PTCP) is a disorder which is characterized by EDTA-dependent anti-platelet autoantibodies that recognize platelet antigens modified with EDTA. Antiplatelet antibodies normally recognize platelet antigens on the EDTA-modified platelet membrane, usually IgG or IgM, but occasionally IgA.

Chelation with EDTA changes the structure of the GPIIb-IIIa platelet membrane complex, exposing an unfamiliar epitope available to autoantibodies. This leads to platelet clumping/in vitro accumulation, that leads to false low platelet counts once automatic detectors are utilized when platelet counts are regular. Platelet clumps due to the elevated white blood cell counts are counted as single giant platelets or as tiny lymphocytes in the white blood cell aperture. The phenomenon takes place both in cases and in healthy individuals with various disorders with a reported incidence of $0.09-0.21 \%[41]$.
In the regulation of inflammation, immune responses, acute phase response, hemopoiesis, inflammation and the central nervous system, IL-6 is a multifunctional cytokine. In nearly every pathophysiological inflammatory condition and autoimmune condition it is expressed primarily and temporarily unregulated. Trans-signaling of IL-6 is also important for disease maintenance, promoting the transition from acute to chronic inflammation. The biological activity of IL- 6 is mediated by two molecules: the IL-6R (IL-6 receptor) and the membrane-bound -receptor glycoprotein 130. (gp130). The signal is transduced through gp130 and through trans-signaling, wherein IL-6 links to soluble forms of the IL-6R. (sIL-6R) (sIL-6R).

Due to the standardized expression of gp130, hypothetically, those agonistic IL-6/sIL-6R combinations will trigger all cells (that is found on all cells). The rest platelets produce gp130, and when IL-6 (generated through pressured endothelial cells) is present, trans-signaling platelet-derived IL- 6 occurs, which may be crucial in inflammation development within a compromised vessel and platelet. Since IL-6 has a universal binding location on all cells, including RBCs, it could affect RBCs the most [42].

$\mathrm{Zn}^{2}$ can enhance heparin-protein interaction in two ways One is achieved by initiating a concomitant change in the protein revealing a latent heparin-binding position, whereas the other uses $\mathrm{Zn}^{2}$ to combine heparin with the protein. Even though $\mathrm{Zn}^{2}$ links to heparin, it can't enhance thrombin or antithrombin reaction with heparin. This indicates that the binding of $\mathrm{Zn}^{2}$ to heparin is protein specific. SPR and fluorescence analysis also indicate that $\mathrm{Zn}^{2}$ causes conformational alterations in fibrinogen. These findings indicate that $\mathrm{Zn}^{2}$ is a frequent mediator of the heparin-protein reaction.

One of the implications of heparin's association with proteins other than antithrombin is a decrease in its anticoagulant function; $\mathrm{Zn}^{2}$ can also increase the thrombogenicity of fibrin thrombi. These findings suggest that several reactions involving $\mathrm{Zn}^{2}$ may have been missed in the plasma due to the use of citrate as an anticoagulant [43].

Platelet factor 4 (heparin neutralizing factor): The presence of pf4 in platelet storage granules was suspected as early as 1948. It is a potent anti-heparin [44]. 
Why Acute Inflammatory Response Can Happen After Adenoviral Vector Vaccines; Is the Interleukin 6 the Secret and How Circumvent that, Is by Adding Polyethylene Glycol (PEG)?

The spleen is known it is the major site of interleukin 6 (IL-6) production, diminished uptake of PEGylated adenoviral vectors by the spleen may explain in part the lower level of serum IL-6 observed after delivery of PEGylated adenoviral vectors, For the first time O'Riordan et al. performed PEGylation of Adenoviral vectors since 1999, Adenoviral vectors have been described to interact with platelets, leading to thrombocytopenia after intravenous delivery [45].

\begin{tabular}{|c|c|c|c|c|}
\hline Vaccine & Pfizer - BioNTech & Moderna & $\begin{array}{l}\text { AstraZeneca vaccine } \\
\text { (ChAd0x1 nCov-19) } \\
\text { (AZD1222) (C19VAZ) } \\
\text { (CoviShield)(Vaxzevria) }\end{array}$ & $\begin{array}{l}\text { Johnson and Johnson (JANSSEN) } \\
\text { covid-19 VACCINE }\end{array}$ \\
\hline Technology & mRNA based & mRNA based & $\begin{array}{l}\text { Adenoviral vector vaccine } \\
\text { (Chimpanzee, ChAdOx1; A } \\
\text { replication incompetent adeno- } \\
\text { virus type }\end{array}$ & $\begin{array}{l}\text { Adenoviral vector vaccine (Ad26.COV2. } \\
\text { S) Single shot (Recombinant replication } \\
\text { incompetent adenovirus type } 26 \text { (Ad26) } \\
\text { vectored vaccine }\end{array}$ \\
\hline $\begin{array}{l}\text { Active com- } \\
\text { ponent }\end{array}$ & $\begin{array}{l}\text { mRNA encoding the } \\
\text { viral spike protein of } \\
\text { SARS-COV2. } \\
\text { ALC-0159 = } \\
\text { 2-[(polyethylene } \\
\text { glycol)-2000]-N,N- } \\
\text { ditetradecylacet- } \\
\text { amide } \\
\text { 1,2-Distearoyl-sn- } \\
\text { glycero-3-phospho- } \\
\text { choline } \\
\text { ALC-0315 = } \\
\text { (4-hydroxybutyl) } \\
\text { azanediyl) bis (hex- } \\
\text { ane-6,1-diyl) bis(2- } \\
\text { hexyldecanoate) } \\
\text { potassium dihydro- } \\
\text { gen phosphate } \\
\text { Potassium chloride } \\
\text { Sodium chloride } \\
\text { Cholesterol } \\
\text { Disodium hydrogen } \\
\text { Phosphate dihydrate } \\
\text { Water for injections } \\
\text { Sucrose [46] }\end{array}$ & $\begin{array}{c}\text { mRNAencod- } \\
\text { ing the viral } \\
\text { spike protein } \\
\text { of SARS-COV2 } \\
\\
\text { Cholesterol } \\
\text { Lipids (SM- } \\
\text { 102, } \\
\text { 1,2-distearoyl- } \\
\text { sn-glycero- } \\
\text { 3-Phosphocho- } \\
\text { line [DSPC]) } \\
\text { Polyethylene } \\
\text { glycol [PEG] } \\
\text { 2000 } \\
\text { 1,2 dimyristoyl } \\
\text { glycerol [DMG] } \\
\text { Acetic acid } \\
\text { Sodium ac- } \\
\text { etate trihy- } \\
\text { drate, } \\
\text { Tromethamine } \\
\text { Hydrochloride } \\
\text { Sucrose } \\
\text { [romethamine }\end{array}$ & $\begin{array}{l}\text { The genetic code for the spike } \\
\text { protein, } \\
\text { Disodium edetate dihydrate } \\
\text { (EDTA, a binding agent). } \\
\text { L-Histidine. } \\
\text { Polysorbate } 80 \\
\text { Magnesium chloride; } \\
\text { Sodium chloride; } \\
\text { Ethanol. } \\
\text { Water for injection (15-16) } \\
\text { Sucrose. } \\
\text { [48] }\end{array}$ & $\begin{array}{c}\text { The genetic code for the spike protein } \\
\text { Ethanol Hydrochloric acid } \\
\text { Polysorbate-80 } \\
\text { 2-hydroxypropyl- } \beta \text {-cyclodextrin (HBCD) } \\
\text { Sodium chloride } \\
\text { Citric acid monohydrate } \\
\text { Trisodium citrate dihydrate } \\
\text { Sodium hydroxide } \\
\text { Water for injections } \\
\text { [49] }\end{array}$ \\
\hline
\end{tabular}

Citation: Amr Ahmed, Hassan Shora, Ahmed Mohamed and Simona Ibrahim. "Why Acute Inflammatory Response Can Happen After Adenoviral Vector Vaccines; Is the Interleukin 6 the Secret and How Circumvent that, Is by Adding Polyethylene Glycol (PEG)?". Acta Scientific Medical Sciences 5.7 (2021): 
Why Acute Inflammatory Response Can Happen After Adenoviral Vector Vaccines; Is the Interleukin 6 the Secret and How Circumvent that, Is by Adding Polyethylene Glycol (PEG)?

\begin{tabular}{|c|c|c|}
\hline $\begin{array}{l}\text { Our } \\
\text { Concerns }\end{array}$ & $\begin{array}{c}\text { The primary concern with } \\
\text { AstraZeneca's excipients is } \\
\text { polysorbate } 80 \text {, that acts as a } \\
\text { stabilizer for the vaccine, and } \\
\text { EDTA, which acts as a binding } \\
\text { agent and a very potent zinc } \\
\text { chelator }\end{array}$ & $\begin{array}{l}\text { here the concern about the excipient } \\
\text { polysorbate } 80\end{array}$ \\
\hline
\end{tabular}

Table 1: The comparison of the 5 vaccine (Under Emergency Use Authorization) according to the active component and inactive ingredients (biologic excipients) of vaccines involved in the acute immune response following vaccine injection: do we have a good comparison among the five vaccines, two of which are adenoviral vector vaccines and two of which are mRNA-based vaccines? According to vaccine technology and excipients.

Finally, the fifth vaccine the Sputnik V vaccine Adenoviral vector vaccine (two human adenoviral vectors (Ad5 and Ad26).

\section{Conclusion}

Adenoviral vector vaccines induce an acute immunogenic response that varies according to the immune status of the individuals injected. The excipient used in the vaccine injection can either increase or decrease the severity of this acute immune response. For example, polyethylene glycol can decrease IL-6 while polysorbate 80 or EDTA can increase severity. In comparison to Americans or Asians, Africans have neutralizing antibodies against the chimpanzee adenoviral vector, which can result in a less acute immunogenic response (less adverse effects). Thus, it is necessary to administer ChAdox1 nCov2 to African populations in order to avoid the vaccine's acute immunogenic impact in neutralizing antibodies existence (vaccine less effective). The question after discussing the acute immune response from adenovirus vectors (IL- 6 is has a role) and Is adding polyethylene glycol to adenoviral vector vaccine lessen the vaccine adverse effects. As modification of Ad vector capsids with synthetic polymers like polyethylene glycol (PEG) has been proven to help in overcoming one of the most complex hurdles of Ad vector gene delivery, namely, the innate immune response to the vector particle.

\section{Bibliography}

1. Bangari DS and Mittal SK. "Development of non-human adenoviruses as vaccine vectors". Vaccine 24 (2006): 849-862.

2. Stephen SL., et al. "Chromosomal integration of adenoviral vector DNA in vivo". Journal of Virology 84 (2010): 9987-9994.
3. Lopez-Gordo E., et al. "Circumventing antivector immunity: potential use of non-human adenoviral vectors". Human Gene Therapy 25.4 (2014): 285-300.

4. Raper SE., et al. "Fatal systemic inflammatory response syndrome in a ornithine transcarbamylase deficient patient following adenoviral gene transfer". Molecular Genetics and Metabolism 80 (2003): 148-158.

5. Lopez-Gordo E., et al. "Circumventing antivector immunity: potential use of non-human adenoviral vectors". Human Gene Therapy 25.4 (2014): 285-300.

6. Morris Susan J., et al. "Simian adenoviruses as vaccine vectors". Future Virology 11.9 (2016): : 649-659.

7. Xiang Z., et al. "Chimpanzee adenovirus antibodies in humans, sub-Saharan Africa". Emerging Infectious Diseases 12.10 (2006): 1596-1599.

8. Tatsis N., et al. "A CD46-binding chimpanzee adenovirus vector as a vaccine carrier". Molecular Therapy 15.3 (2007): 608-617.

9. Draper SJ and Heeney JL. "Viruses as vaccine vectors for infectious diseases and cancer". Nature Reviews Microbiology 8 (2010): : 62-73.

10. Dicks Matthew DJ., et al. "A Novel Chimpanzee Adenovirus Vector with Low Human Seroprevalence: Improved Systems for Vector Derivation and Comparative Immunogenicity". Plos One 7.7 (2012): :e40385.

11. Dicks MD., et al. "A novel chimpanzee adenovirus vector with low human seroprevalence: improved systems for vector deri- 
Why Acute Inflammatory Response Can Happen After Adenoviral Vector Vaccines; Is the Interleukin 6 the Secret and How Circumvent that, Is by Adding Polyethylene Glycol (PEG)?

vation and comparative immunogenicity". Plos One 7 (2012): : e40385.

12. AstraZeneca. "COVID-19 Vaccine Product Monograph". Astrazeneca Canada Inc (2021).

13. Richard D Antrobus., et al. "The Jenner Institute, University of Oxford, Oxford, UK". Molecular Therapy 22.3 (2014): 668-674.

14. Shimizu K., et al. "Quantitative analysis of the leaky expression of adenovirus genes in cells transduced with a replicationincompetent adenovirus vector". Molecular Pharmaceutics 8 (2011): :1430-1435.

15. Catherine M Bollard., et al. (2010).

16. Ha E., et al. "Peroxide formation in polysorbate 80 and protein stability". Journal of Pharmaceutical Sciences 91.10 (2002): 2252-2264.

17. Singh SR., et al. "Effect of Polysorbate 80 Quality on Photostability of a Monoclonal Antibody". AAPS PharmSciTech 13.2 (2012): 422-430.

18. Mok H., et al. "Evaluation of polyethylene glycol modification of first-generation and helper-dependent adenoviral vectors to reduce innate immune responses". Molecular Therapy 11 (2005): 66-79.

19. Appledorn DM., et al. "Complex interactions with several arms of the complement system dictate innate and humoral immunity to adenoviral vectors". Gene Therapy 15 (2008): :16061617.

20. Ahi YS., et al. "Adenoviral vector immunity: its implications and circumvention strategies". Current Gene Therapy 11.4 (2011): 307-320.

21. Wolins N., et al. "Intravenous administration of replication-incompetent adenovirus to rhesus monkeys induces thrombocytopenia by increasing in vivo platelet clearance". British Journal of Haematology 123 (2003): :903-905.

22. Peng Y., et al. "Inhibition of tumor necrosis factor alpha by an adenovirus-encoded soluble fusion protein extends transgene expression in the liver and lung". Journal of Virology 73 (1999): 5098-5109.
23. Ahi YS., et al. "Adenoviral vector immunity: its implications and circumvention strategies". Current Gene Therapy 11.4 (2011): 307-320.

24. Appledorn DM., et al. "Complex interactions with several arms of the complement system dictate innate and humoral immunity to adenoviral vectors". Gene Therapy 15.24 (2008): 16061617.

25. Cichon G., et al. "Complement activation by recombinant adenoviruses". Gene Therapy 8.23 (2001): 1794-1800.

26. Mok H., et al. "Microencapsulation of PEGylated adenovirus within PLGA microspheres for enhanced stability and gene transfection efficiency". Pharmaceutical Research 24.12 (2007): 2263-2269.

27. De Geest B., et al. "Elimination of innate immune responses and liver inflammation by PEGylation of adenoviral vectors and methylprednisolone". Human Gene Therapy 16.12 (2005): 1439-1451.

28. Sailaja G., et al. "Encapsulation of recombinant adenovirus into alginate microspheres circumvents vector-specific immune response". Gene Therapy 9.24 (2002): 1722-1729.

29. Xiang Z., et al. "Chimpanzee adenovirus antibodies in humans, sub-Saharan Africa". Emerging Infectious Diseases 12.10 (2006): 1596-1599.

30. Engelhardt JF., et al. "Ablation of E2A in recombinant adenoviruses improves transgene persistence and decreases inflammatory response in mouse liver". Proceedings of the National Academy of Sciences of the United States of America 91 (1994): 6196-200.

31. Lopez-Gordo E., et al. "Circumventing antivector immunity: potential use of non-human adenoviral vectors". Human Gene Therapy 25.4 (2014): 285-300.

32. Wold WS and Toth K. "Adenovirus vectors for gene therapy, vaccination and cancer gene therapy". Current Gene Therapy 13.6 (2013): 421-433.

33. Reid T., et al. "Intravascular adenoviral agents in cancer patients: lessons from clinical trials". Cancer Gene Therapy 9 (2002): 979-986. 
34. Nyborg JK and Peersen OB. "That zincing feeling: the effects of EDTA on the behaviour of zinc-binding transcriptional regulators". Biochemistry Journal 381 (2004): e3-e4.

35. Chun-Hui Fang., et al. "EDTA-dependent pseudothrombocytopenia”. Formosan Journal of Surgery 48.3 (2015): 107-109.

36. Ben R Watson., et al. "Zinc is a transmembrane agonist that induces platelet activation in a tyrosine phosphorylation-dependent manner". Metallomics 8.1 (2016): 91-100.

37. Elena Y Senchenkova., et al. "Novel Role of T Cells and IL-6 (Interleukin-6) in Angiotensin II-Induced Microvascular Dysfunction". Hypertension 73 (2019): 829-838.

38. Aymeric A Amelot., et al. "Platelet Factor 4 (CXCL4) Seals Blood Clots by Altering the Structure of Fibrin". 282.1 (2007): 710-720.

39. Käser-Glanzmann R., et al. "Heparin-Neutralizing Factor (Platelet Factor 4) from Human Blood Platelets and its Reactivity with Fibrinogen and Soluble Fibrin-Monomer Complexes". Pathophysiology of Haemostasis and Thrombosis 1 (1972): 136-147.

40. Dr Ron Kerr. Clinical Lecturer, Department of Haematology, The Royal Infirmary of Edinburgh, 1 Lauriston Place, Edinburgh EH3 9YW UK.

41. Pitkin. Journal of Traditional Medicine and Clinical Naturopathy 6 (2017): 1.

42. Bester J and Pretorius E. "Effects of IL-1 $\beta$, IL-6 and IL-8 on erythrocytes, platelets and clot viscoelasticity". Scientific Report 6 (2016): 32188.

43. James C Fredenburgh., et al. "Mediates High Affinity Binding of Heparin to the C Domain of Fibrinogen". Journal of Biological Chemistry 288.41 (2013): 29394-29402.

44. Shirley P Levine and Herbert Wohl. "Human platelet factor 4: Purification and characterization by affinity chromatography". Purification of human platelet factor 4 .

45. Kreppel F and Kochanek S. "Modification of adenovirus gene transfer vectors with synthetic polymers: a scientific review and technical guide". Molecular Therapy 16.1 (2008): 16-29.
46. https://ec.europa.eu/health/documents/communityregister/2020/20201221150522/anx_150522_en

47. https://www.fda.gov/media/144638/download

48. https://www.ema.europa.eu/en/documents/product-information/covid-19-vaccine-astrazeneca-product-informationapproved-chmp-29-january-2021

49. https://www.fda.gov/media/146217/download

50. Wilson JM. "Lessons learned from the gene therapy trial for ornithine transcarbamylase deficiency". Molecular Genetics and Metabolism 96 (2006): :151-157.

\section{Volume 5 Issue 7 July 2021 \\ (C) All rights are reserved by Amr Ahmed., et al.}

